\title{
Circular chromatic number of planar graphs of large odd girth
}

\author{
Xuding Zhu* \\ Department of Applied Mathematics \\ National Sun Yat-sen University \\ Kaohsiung, Taiwan 80424 \\ zhu@math.nsysu.edu.tw
}

Submitted: October 17, 2000; Accepted: June 7, 2001.

Mathematical Subject Classification: 05C15

\begin{abstract}
It was conjectured by Jaeger that $4 k$-edge connected graphs admit a $(2 k+$ $1, k)$-flow. The restriction of this conjecture to planar graphs is equivalent to the statement that planar graphs of girth at least $4 k$ have circular chromatic number at most $2+\frac{1}{k}$. Even this restricted version of Jaeger's conjecture is largely open. The $k=1$ case is the well-known Grötzsch 3-colour theorem. This paper proves that for $k \geq 2$, planar graphs of odd girth at least $8 k-3$ have circular chromatic number at most $2+\frac{1}{k}$.
\end{abstract}

\section{Introduction}

Let $G$ be a graph and $D$ an orientation of $G$. For positive integers $k \geq 2 d$, a $(k, d)$-flow $f$ of $D$ is a mapping that assigns to each edge $e$ of $D$ an integer $f(e)$ such that (i): for every vertex $x, \Sigma_{e \in E^{+}(x)} f(e)-\Sigma_{e \in E^{-}(x)} f(e)=0$, and (ii) for every edge $e, d \leq|f(e)| \leq k-d$. Here $E^{+}(x)$ is the set of edges incident to and oriented away from $x$, and $E^{-}(x)$ is the set of edges incident to and oriented towards $x$. A graph $G$ is said to admit a $(k, d)$-flow if $G$ has an orientation $D$ that admits a $(k, d)$-flow. The following conjecture was proposed by Jaeger $[7,8]$ :

Conjecture 1.1 For any integer $k \geq 1$, every $4 k$-edge-connected graph admits an $(2 k+$ $1, k)$-flow.

\footnotetext{
${ }^{*}$ This research was partially supported by the National Science Council under grant NSC89-2115-M$110-012$
} 
Jaeger's conjecture is very strong. The $k=1$ case is Tutte's 3 -flow conjecture [16], and the $k=2$ case implies Tutte's 5-flow conjecture [17]. Both the 3-flow conjecture and the 5-flow conjecture are long standing open problems [20]. Many difficult conjectures for flows have been proved for planar graphs. However, even restricted to planar graphs, Jaeger's conjecture remains largely open.

For planar graphs, the flow problem can be dualized to a colouring problem. For positive integers $k \geq 2 d$, a $(k, d)$-colouring of a graph $G$ is a mapping $f: V(G) \rightarrow$ $\{0,1, \cdots, k-1\}$ such that $d \leq|f(x)-f(y)| \leq k-d$ for every edge $x y$ of $G$. The circular chromatic number $\chi_{c}(G)$ of $G$ is defined as

$$
\chi_{c}(G)=\min \{k / d: \text { there exists a }(k, d) \text {-colouring of } G\} .
$$

The circular chromatic number (also known as the "star chromatic number" [18]) has been studied extensively in the past decade. Readers are referred to [22] for a survey on this subject. A $(k, d)$-colouring of a planar graph $G$ corresponds to a $(k, d)$-flow of the dual graph of $G$ [4], [22]. Therefore, the restriction of Jaeger's conjecture to planar graphs is equivalent to the following:

Conjecture 1.2 [Jaeger's conjecture restricted to planar graphs] Every planar graph $G$ of girth at least $4 k$ has circular chromatic number at most $2+\frac{1}{k}$.

It was proved by Galluccio, Goddyn and Hell [3] that for each $\epsilon>0$, for every surface $S$, there exists an integer $g$ such that every graph of girth at least $g$ embedded in $S$ has circular chromatic number at most $2+\epsilon$. In particular, we seek the smallest integer $g(k)$ such that every planar graph $G$ of girth at least $g(k)$ has $\chi_{c}(G) \leq 2+\frac{1}{k}$. It follows from Grötzsch's Theorem (triangle free planar graphs are 3-colourable [5]) that $g(1)=4$. For $k \geq 2$, the best known bounds at present are $4 k \leq g(k) \leq 10 k-4$. The upper bound was proved by Galluccio, Goddyn and Hell [3], and the lower bound was proved by DeVos [2]. Conjecture 1.2 asserts that the lower bound is tight.

The odd edge-connectivity of a graph $G$ is the size of a smallest odd edge cut of $G$. The odd girth of $G$ is the length of a shortest odd cycle of $G$. Zhang [19] proposed a strengthing of Jaeger's conjecture, where the edge-connectivity condition is replaced by an odd edgeconnectivity condition. Conjecture 1.3 below is the restriction of that conjecture to planar graphs (in the dual version).

Conjecture 1.3 [19] Every planar graph $G$ of odd girth at least $4 k+1$ has circular chromatic number at most $2+\frac{1}{k}$.

It was proved by Klostermeyer and Zhang that for any $\epsilon>0$, there is an integer $f$ such that every planar graph of odd girth at least $f$ has circular chromatic number at most $2+\epsilon$. We seek the smallest odd integer $f(k)$ such that every planar graph of odd girth at least $f(k)$ has circular chromatic number at most $2+\frac{1}{k}$. The best known bounds at present are $4 k+1 \leq f(k) \leq 10 k-3$. The upper bound was proved by Klostermeyer and Zhang, and lower bound follows from the lower bound for $g(k)$. 
It follows from the definitions that $f(k) \geq g(k)+1$. So Conjecture 1.3 is stronger than Conjecture 1.2. In this paper, we improve the upper bound for $f(k)$, which also yields a better upper bound for $g(k)$.

Theorem 1.4 For any integer $k \geq 2$, every planar graph $G$ of odd girth at least $8 k-3$ has circular chromatic number at most $2+\frac{1}{k}$.

Note that Theorem 1.4 remains true for $k=1$. The $k=1$ case is exactly the Grötzsch 3 -colour theorem [5]. However, the proof presented here does not work for this case.

The proof uses the discharging method. In Section 2, we shall give a family of unavoidable configurations in a counterexample of Theorem 1.4. In Section 3, we prove that any graph containing one of the unavoidable configurations cannot be a minimum counterexample.

\section{Unavoidable configurations}

Let $G$ be a graph. A thread in $G$ is a maximal subgraph of $G$ which is a path whose internal vertices all have degree 2 in $G$. For $t \geq 1$, vertices $x$ and $y$ are loosely $t$-adjacent (or loosely adjacent if $t$ is irrelevant) if $G$ contains an $x, y$-path $P$ of length $t$ lies in a thread, i.e., $P$ is a path whose internal vertices all have degree 2 in $G$. Let $d_{l}(x)$ be the number of vertices that are loosely adjacent to $x$, and let $d(x)$ the degree of $x$.

Lemma 2.1 Let $G$ be a planar graph of minimum degree at least 2. If each facial cycle of $G$ has length at least $8 k-3$, then one of the following holds:

(a) G has a vertex $x$ such that $d_{l}(x) \geq 2 k(d(x)-1)$.

(b) $G$ has two loosely $t$-adjacent vertices $x$ and $y$ such that $d(x)=d(y)=3$ and $d_{l}(x)+d_{l}(y) \geq 6 k+t$.

(c) $G$ has a vertex $x$ of degree 3 which is loosely adjacent to $a, b, c$ such that $d(a)=$ $d(b)=d(c)=3$ and $d_{l}(a)=d_{l}(b)=d_{l}(c)=4 k-1$.

Proof. The proof uses the discharging method. Assume that $G$ is a counterexample to Lemma 2.1. We first assign a charge $c(x)$ to each vertex $x$ and prove that the total charge $\sum_{x \in V(G)} c(x)$ is negative. Then by two rounds of discharging, each vertex $x$ gets a new charge $c^{*}(x)$. We prove that the total charge is unchanged and yet $c^{*}(x) \geq 0$ for each vertex $x$. This is an obvious contradiction, which shows that the counterexample $G$ does not exist.

Since $G$ is a counterexample, condition (a) does not hold. This implies that $G$ has no thread of length at least $2 k$. For otherwise, for any internal vertex $x$ of a thread of length 
at least $2 k$, we have $d_{l}(x) \geq 2 k=2 k(d(x)-1)$. Thus we may assume that each cycle of $G$ contains at least 3 vertices of degree at least 3 (otherwise $G$ contains a cycle which contains at most two vertices of degree at least 3. By appropriately embed the graph, we may assume this cycle is a facial cycle, which then must have length at least $8 k-3$. Hence $G$ has a thread of length at least $2 k$ ).

Let $v, e, f$ be the numbers of vertices, edges and faces of $G$ respectively. Since each facial cycle of $G$ has length at least $8 k-3$, and the sum of the lengths of all the facial cycles is equal to $2 e$, it follows that $(8 k-3) f \leq 2 e$. Plugging this into Euler's formula $v+f-e=2$, we have

$$
(8 k-5) e \leq(8 k-3)(v-2)<(8 k-3) v .
$$

Assign to each vertex $x$ of $G$ a charge

$$
c(x)=(8 k-5) d(x)-(16 k-6) .
$$

The total charge assigned to the vertices of $G$ is

$$
\begin{aligned}
\Sigma_{x \in V(G)} c(x) & =\Sigma_{x \in V(G)}((8 k-5) d(x)-(16 k-6)) \\
& =2 e(8 k-5)-(16 k-6) v \\
& <2(8 k-3) v-(16 k-6) v \\
& =0 .
\end{aligned}
$$

Discharging rule for the first round: Transfer a charge of amount 2 from each vertex $x$ of degree at least 3 to each vertex $y$ of degree 2 that is loosely adjacent to $x$.

If $y$ has degree 2 , then $y$ is loosely adjacent to two vertices of degree at least 3 . Thus the total amount of charge received by $y$ is 4 . So the new charge $c^{\prime}(y)$ at $y$ is

$$
c^{\prime}(y)=2(8 k-5)-(16 k-6)+4=0 .
$$

By definition, each vertex $x$ is loosely adjacent to $d(x)$ vertices of degree at least 3 . So $x$ is loosely adjacent to $d_{l}(x)-d(x)$ vertices of degree 2 . As condition (a) does not hold, $d_{l}(x) \leq 2 k(d(x)-1)-1$. If $d(x) \geq 3$, then the total amount of charge sent out from $x$ is

$$
2\left(d_{l}(x)-d(x)\right) \leq(4 k-2) d(x)-(4 k+2) .
$$

If $d(x) \geq 4$, then the new charge $c^{\prime}(x)$ at $x$ is

$$
\begin{aligned}
c^{\prime}(x) & \geq d(x)(8 k-5)-(16 k-6)-((4 k-2) d(x)-(4 k+2)) \\
& =d(x)(4 k-3)-(12 k-8) \\
& =d(x)+d(x)(4 k-4)-(12 k-8) \\
& \geq d(x) .
\end{aligned}
$$

If $x$ has degree 3 , then as condition (a) does not hold, $d_{l}(x) \leq 4 k-1$. The same calculation shows the following: 
- If $d_{l}(x)=4 k-1$, then $c^{\prime}(x)=-1$.

- If $d_{l}(x)=4 k-2$, then $c^{\prime}(x)=1$.

- If $d_{l}(x) \leq 4 k-3$, then $c^{\prime}(x) \geq 3$.

We call a vertex $x$ critical if $d(x)=3$ and $d_{l}(x)=4 k-1$. The critical vertices are the only vertices having negative charge at this moment. We apply a second round of discharging as follows:

Discharging rule for the second round: Suppose $x$ has degree at least 4 or $x$ has degree 3 and $d_{l}(x) \leq 4 k-3$. If $x$ is loosely adjacent to a critical vertex $y$, then transfer a charge of amount 1 from $x$ to $y$. Suppose $x$ has degree 3 and $d_{l}(x)=4 k-2$. If $x$ is loosely adjacent to a critical vertex $y$, then transfer a charge of amount $\frac{1}{2}$ from $x$ to $y$.

The new charge at vertex $x$ after the second round of discharging is denoted by $c^{*}(x)$. We shall prove $c^{*}(x) \geq 0$ for all $x$.

If $x$ has degree 2 , then $c^{*}(x)=c^{\prime}(x)=0$.

If $d(x) \geq 4$, then $c^{\prime}(x) \geq d(x)$. There are at most $d(x)$ critical vertices loosely adjacent to $x$, so the amount of charge sent out from $x$ (at the second round) is at most $d(x)$. Therefore $c^{*}(x) \geq 0$.

If $x$ has degree 3 and $d_{l}(x) \leq 4 k-3$, then $c^{\prime}(x) \geq 3$. The amount of charge sent out from $x$ (at the second round) is at most 2. Therefore $c^{*}(x) \geq 0$.

Suppose $x$ has degree 3 and $d_{l}(x)=4 k-2$. Then $c^{\prime}(x)=1$. As condition (c) does not hold, there are at most two critical vertices that are loosely adjacent to $x$. Hence the amount of charge sent out from $x$ (at the second round) is at most 1 . Therefore $c^{*}(x) \geq 0$.

It remains to consider critical vertices. If a critical vertex $w$ is loosely adjacent to a vertex of degree at least 4 or loosely adjacent to a vertex $a$ with $d(a)=3$ and $d_{l}(a) \leq 4 k-3$, then $w$ receives at least an amount 1 of charge in the second round of discharging. Hence $c^{*}(w) \geq c^{\prime}(w)+1=0$.

Assume that $w$ is loosely adjacent to three vertices of degree 3, say $a, b, c$, such that $d_{l}(a), d_{l}(b), d_{l}(c) \geq 4 k-2$. Suppose $w$ is loosely $t_{1}$-adjacent to $a$, loosely $t_{2}$-adjacent to $b$ and loosely $t_{3}$-adjacent to $c$. Then $d_{l}(w)=t_{1}+t_{2}+t_{3}=4 k-1$. Since $G$ is a counterexample, condition (b) does not hold, i.e., $G$ does not have two loosely $t$-adjacent vertices $x$ and $y$ such that $d(x)=d(y)=3$ and $d_{l}(x)+d_{l}(y) \geq 6 k+t$. Therefore

$$
\begin{aligned}
& d_{l}(a)+d_{l}(w) \leq 6 k+t_{1}-1, \\
& d_{l}(b)+d_{l}(w) \leq 6 k+t_{2}-1, \\
& d_{l}(c)+d_{l}(w) \leq 6 k+t_{3}-1 .
\end{aligned}
$$

As $d_{l}(w)=4 k-1$ and $d_{l}(a), d_{l}(b), d_{l}(c) \geq 4 k-2$, we conclude that $t_{i} \geq(4 k-2)+(4 k-$ $1)-(6 k-1)=2 k-2 \geq 2$ for $i=1,2,3$. Hence $4 k-1=t_{1}+t_{2}+t_{3} \geq 6 k-6$, which implies that $k=2$. As $t_{i}<2 k=4$, we have $2 \leq t_{i} \leq 3$. Since $t_{1}+t_{2}+t_{3}=7$, without loss of generality, we may assume that $t_{1}=3, t_{2}=t_{3}=2$. Now $d_{l}(b)+d_{l}(w) \leq 13$ implies 
that $d_{l}(b) \leq 6$, and hence $d_{l}(b)=6$. Similarly, $d_{l}(c)=6$. By our discharging rule, each of $b$ and $c$ transfer an amount of $\frac{1}{2}$ of charge to $w$. Hence $c^{*}(w)=c^{\prime}(w)+1=0$.

So each vertex $x$ of $G$ has a nonnegative new charge $c^{*}(x)$, contrary to the fact that the total charge is negative.

\section{Reducibility of the unavoidable configurations}

This section proves Theorem 1.4, by showing that any minimal counterexample does not contain any of those unavoidable configurations listed in Lemma 2.1. In the remainder of this section, $k \geq 2$ is a fixed integer, and we consider only $(2 k+1, k)$-colouring of graphs. Thus the colour set is $C=\{0,1,2, \cdots, 2 k\}$.

First we consider extending partial colourings of paths. Let $P$ be an $x, y$-path of length $n$. Let $S$ be a set of colours. Let $\phi(n, S)=\{j \in C$ : there exists a $(2 k+1, k)$-colouring $f$ of $P$ such that $f(x) \in S$ and $f(y)=j$ \}.

Lemma 3.1 For any nonempty subset $S$ of $C,|\phi(n, S)| \geq \min \{2 k+1,|S|+n\}$.

Proof. It suffices to prove the claim for $n=1$, since applying that for each successive edge yields the full statement. Let $S^{\prime}=\phi(1, S)$. We define an auxiliary bipartite graph $H$. Introduce a vertex $a_{i}$ for each $i \in S$ and a vertex $b_{j}$ for each $j \in S^{\prime}$. Let $a_{i} b_{j}$ be an edge if and only if there is a $(2 k+1, k)$-colouring $f$ of the edge $x y$ such that $f(x)=i$ and $f(y)=j$. In $H$, each $a_{i}$ has degree $2\left(\right.$ if $i \in S$, then $\left.i+k \bmod (2 k+1), i+k+1 \bmod (2 k+1) \in S^{\prime}\right)$, and each $b_{j}$ has degree at most 2 . Moreover, $b_{j}$ has degree 2 for all $j \in S^{\prime}$ only if $S^{\prime}=C$. Therefore $\left|S^{\prime}\right| \geq \min \{2 k+1,|S|+1\}$.

Corollary 3.2 Let $P_{1}, P_{2}, \cdots, P_{n}$ be paths that are pairwise disjoint except for one common endpoint $y$. Let $x_{i}$ and $t_{i}$ be the other endpoint and the length of $P_{i}$. If $2(j-$ $1) k \leq \Sigma_{i=1}^{j} t_{i} \leq 2 j k$ for $j=2,3, \cdots, n$, then any $(2 k+1, k)$-colouring $f$ of the vertices $x_{1}, x_{2}, \cdots, x_{k}$ can be extended to a $(2 k+1, k)$-colouring of $\cup_{i=1}^{n} P_{i}$.

Proof. For $i=1,2, \cdots, n$, let $S_{i}=\left\{j\right.$ : there exists a $(2 k+1, k)$-colouring $g$ of $P_{i}$ such that $g\left(x_{i}\right)=f\left(x_{i}\right)$ and $\left.g(y)=j\right\}$. By Lemma 3.1, $\left|S_{i}\right| \geq 1+t_{i}$. Obviously $f$ can be extended to a $(2 k+1, k)$-colouring of $\cup_{i=1}^{n} P_{i}$ if and only if $S_{1} \cap S_{2} \cap \cdots \cap S_{n} \neq \emptyset$. Since $\left|S_{1} \cap S_{2}\right|=\left|S_{1}\right|+\left|S_{2}\right|-\left|S_{1} \cup S_{2}\right|$ and $\left|S_{1} \cup S_{2}\right| \leq 2 k+1$, we know that $\left|S_{1} \cap S_{2}\right| \geq t_{1}+t_{2}+1-2 k$, where by assumption $1 \leq t_{1}+t_{2}+1-2 k \leq 2 k+1$. By induction on $i$, it follows that

$$
\left|S_{1} \cap S_{2} \cap \cdots \cap S_{i}\right| \geq t_{1}+t_{2}+\cdots+t_{i}+1-2(i-1) k,
$$

and

$$
1 \leq t_{1}+t_{2}+\cdots+t_{i}+1-2(i-1) k \leq 2 k+1
$$


Lemma 3.3 If $G$ has a vertex $x$ such that $d_{l}(x) \geq 2 k(d(x)-1)$, then $G$ has proper subgraph $G^{\prime}$ such that any $(2 k+1, k)$-colouring of $G^{\prime}$ can be extended to a $(2 k+1, k)$ colouring of $G$.

Proof. Assume that $x$ is a vertex of $G$ with $d_{l}(x) \geq 2 k(d(x)-1)$. If $d(x)=2$, then $x$ is the internal vertex of a thread $P$ of length at least $2 k$. Let $G^{\prime}$ be obtained from $G$ by deleting the internal vertices of $G$. Corollary 3.2 implies that any $(2 k+1, k)$-colouring of $G^{\prime}$ can be extended to a $(2 k+1, k)$-colouring of $G$. Thus we may assume that $d(x)=n \geq 3$. Let $P_{1}, P_{2}, \cdots, P_{n}$ be the $n$ threads of $G$ ending at $x$. Let $t_{i}$ be the length of $P_{i}$. Then $t_{1}+t_{2}+\cdots+t_{n}=d_{l}(x) \geq 2 k(n-1)$. Since each thread of $G$ has length at most $2 k-1$, we have $2 k(j-1) \leq t_{1}+t_{2}+\cdots+t_{j} \leq 2 k j$ for $j=2,3, \cdots, n$,. Let $G^{\prime}$ be obtained from $G$ by deleting $x$ and the internal vertices of $P_{1}, P_{2}, \cdots, P_{n}$. The conclusion follows from Corollary 3.2 .

Lemma 3.4 Assume $x$ and $y$ are two loosely t-adjacent vertices of $G$ of degree 3 . If $d_{l}(x)+d_{l}(y) \geq 6 k+t$, then there is a proper subgraph $G^{\prime}$ of $G$ such that any $(2 k+1, k)$ colouring of $G^{\prime}$ can be extended to a $(2 k+1, k)$-colouring of $G$.

Proof. We may assume that $G$ has no thread of length at least $2 k$. Let $P$ be the thread of length $t$ joining $x$ and $y$. Since $d(x)=3$, we may let $x_{1}$ and $x_{2}$ be vertices of degree at least 3 other than $y$ that are loosely adjacent to $x$, with $P_{1}$ and $P_{2}$ being the threads joining them to $x$. Similarly, let $y$ be loosely adjacent to vertices $y_{1}$ and $y_{2}$ of degree at least 3 via threads $P_{3}$ and $P_{4}$ other than $P$. Let $G^{\prime}$ be obtained from $G$ by deleting $x, y$ and the internal vertices of $P_{1}, P_{2}, P, P_{3}, P_{4}$. We shall prove that any $(2 k+1, k)$-colouring $f$ of $G^{\prime}$ can be extended to $G$.

Suppose $P_{i}$ has length $t_{i}$. Since $t_{1}+t_{2}+t+t_{3}+t_{4} \geq 6 k$ and $G$ contains no thread of length at least $2 k$, it follows that $t_{1}+t_{2} \geq 2 k+1$ and $t_{3}+t_{4} \geq 2 k+1$. Let $S=\{j$ : there is an extension $g$ of $f$ to $P_{1} \cup P_{2}$ such that $\left.g(x)=j\right\}$. By Corollary $3.2,|S| \geq$ $t_{1}+t_{2}+1-2 k>0$. Let $S^{\prime}=\left\{j\right.$ : there is an extension $g$ of $f$ to $P_{1} \cup P_{2} \cup P$ such that $g(y)=j\}$. By Lemma 3.1, $\left|S^{\prime}\right| \geq t_{1}+t_{2}+t+1-2 k$. Let $S^{\prime \prime}=\{j:$ there is an extension $g$ of $f$ to $P_{3} \cup P_{4}$ such that $\left.g(y)=j\right\}$. By Corollary $3.2,\left|S^{\prime \prime}\right| \geq t_{3}+t_{4}+1-2 k$. Obviously, $f$ can be extended to $G$ if and only if $S^{\prime} \cap S^{\prime \prime} \neq \emptyset$. This is so because $\left|S^{\prime}\right|+\left|S^{\prime \prime}\right| \geq t_{1}+t_{2}+t+1-2 k+t_{3}+t_{4}+1-2 k \geq 2 k+2$.

Lemma 3.5 Suppose $G$ has a vertex $x$ of degree 3 which is loosely adjacent to three critical vertices $a, b, c$. Then $G$ has a proper subgraph $G^{\prime}$ such that any $(2 k+1, k)$-colouring $f$ of $G^{\prime}$ can be extended to a $(2 k+1, k)$-colouring of $G$.

Proof. Let $P_{1}, P_{2}, \cdots, P_{9}$ be the threads incident to $a, b, c$ as shown in Figure 1, where $x_{1}, x_{2}, y_{1}, y_{2}, z_{1}, z_{2}$ are vertices of degree at least 3 .

Let $G^{\prime}$ be obtained from $G$ by deleting $x, a, b, c$ and the internal vertices of $P_{1}, \cdots, P_{9}$. Let $f$ be a $(2 k+1, k)$-colouring of $G^{\prime}$. Let $S_{1}=\{j$ : there is an extension $g$ of $f$ to $P_{1} \cup P_{2} \cup P_{3}$ such that $\left.g(x)=j\right\}, S_{2}=\left\{j\right.$ : there is an extension $g$ of $f$ to $P_{4} \cup P_{5} \cup P_{6}$ 


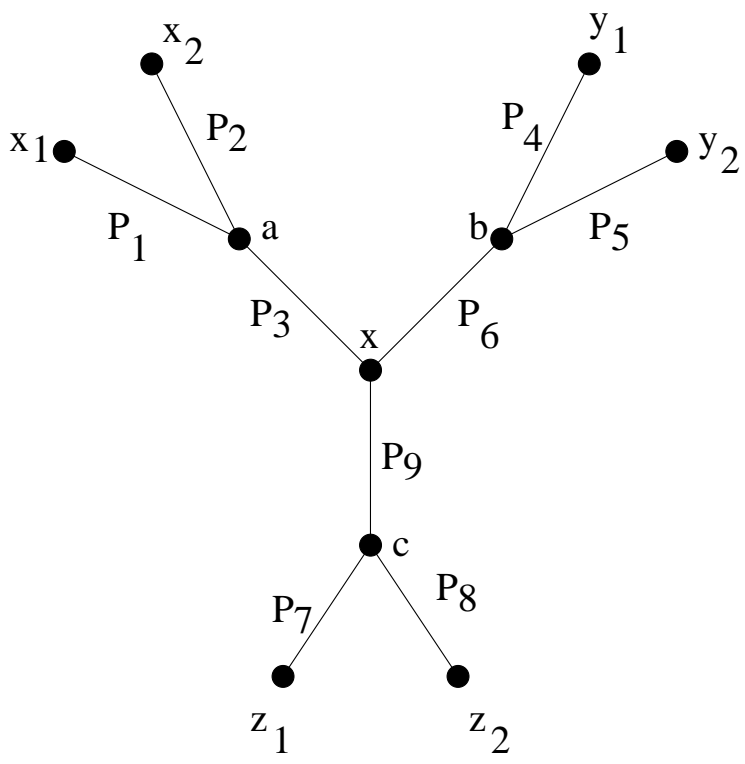

Figure 1: Threads incident to $a, b, c$

such that $g(x)=j\}, S_{3}=\left\{j\right.$ : there is an extension $g$ of $f$ to $P_{7} \cup P_{8} \cup P_{9}$ such that $g(x)=j$. By the argument as in the proof of Lemma 3.4 (see the calculation of $\left|S^{\prime}\right|$ ), $\left|S_{i}\right| \geq 2 k$. It follows that $S_{1} \cap S_{2} \cap S_{3} \neq \emptyset$. Therefore $f$ can be extended to $G$.

To complete the proof of Theorem 1.4, we need one more lemma (the Folding Lemma) from $[9]$.

Lemma 3.6 [9] Suppose $G$ is a planar graph of odd girth at least $8 k-3$. Then either every facial cycle of $G$ has length at least $8 k-3$, or there exists a planar graph $G^{\prime}$ such that

- $\left|V\left(G^{\prime}\right)\right|<|V(G)|$.

- $G$ admits a homomorphism to $G^{\prime}$.

- $G^{\prime}$ has odd girth at least $8 k-3$ and, moreover, each facial cycle of $G^{\prime}$ has length at least $8 k-3$.

Lemma 3.6 says that if $G$ has a facial cycle of small length (which must be even), then that face can be "folded" without creating short odd cycles.

Proof of Theorem $\mathbf{1 . 4}$ If the claim does not hold, we may consider a smallest counterexample $G$. By Lemma 3.6, we may assume that each facial cycle of $G$ has length at least $8 k-3$. By Lemma 2.1, one of the following holds:

- $G$ has a vertex $x$ such that $d_{l}(x) \geq 2 k(d(x)-1)$. 
- $G$ has two loosely $t$-adjacent vertices $x$ and $y$ such that $d(x)=d(y)=3$ and $d_{l}(x)+d_{l}(y) \geq 6 k+t$.

- $G$ has a vertex $x$ of degree 3 which is loosely adjacent to three critical vertices.

Since $G$ is a minimal counterexample, any proper subgraph of $G$ is $(2 k+1, k)$ colourable. However, by Lemmas 3.3, 3.4, and 3.5, in each of the above cases $G$ has a proper subgraph $G^{\prime}$ such that any $(2 k+1, k)$-colouring of $G^{\prime}$ can be extended to a $(2 k+1, k)$-colouring of $G$. This is a contradiction.

Corollary 3.7 If $G$ is a planar graph of girth at least $8 k-4$, then $\chi_{c}(G) \leq 2+\frac{1}{k}$.

\section{A remark on the relation between $g(k)$ and $f(k)$}

We observed earlier that it follows from the definition that $f(k) \geq g(k)+1$. It is unknown if equality holds for all $k$.

Question 4.1 For the functions $g(k), f(k)$ defined above, is it true that $f(k)=g(k)+1$ ?

There is some evidence supporting a positive answer. (Also, Conjecture 1.3 implies a positive answer). Firstly, all the presently known methods for proving upper bounds for $g(k)$ are based on Euler's formula. The girth requirement is only used in the sense that every facial cycle has length at least $g(k)$. By the "folding lemma" of Klostermeyer and Zhang [9], if a planar graph $G$ has odd girth at least $f(k)$, then (for the purpose of investigating circular chromatic number) we may assume that it has large facial cycles as well (cf. the proof of Theorem 1.4).

Secondly, based on a good understanding of the relationship between the circular chromatic number and the girth (as well as the odd girth) of series-parallel graphs [1, $6,11,12]$, we can show that the analogue of Conjecture 4.1 for series-parallel graphs is true. (Series-parallel graphs are graphs obtained from $K_{2}$ by repeatedly applying two operations: subdividing an edge and duplicating an edge.) The following results were proved by Pan and Zhu $[11,12]$.

Theorem 4.2 [11] Suppose $G$ is a series-parallel graph. If $G$ has odd girth at least $6 k-1$, then $\chi_{c}(G) \leq 8 k /(4 k-1)$. If $G$ has odd girth at least $6 k+1$, then $\chi_{c}(G) \leq(4 k+1) / 2 k$. If $G$ is has odd girth at least $6 k+3$, then $\chi_{c}(G) \leq(4 k+3) /(2 k+1)$.

Theorem 4.3 [12] Let $k \geq 1$ be an integer, and let $\epsilon>0$. There exists a series-parallel graph $G$ of girth $6 k-1$ with $\chi_{c}(G)>8 k /(4 k-1)-\epsilon$. There exists a series-parallel graph $G$ of girth $6 k+1$ with $\chi_{c}(G)>(4 k+1) / 2 k-\epsilon$. There exists a series-parallel graph $G$ of girth $6 k+3$ with $\chi_{c}(G)>(4 k+3) /(2 k+1)-\epsilon$. 
For any $0<\epsilon<1$, let $g^{*}(\epsilon)$ be the smallest integer such that every series-parallel graph $G$ of girth at least $g^{*}(\epsilon)$ has $\chi_{c}(G) \leq 2+\epsilon$; let $f^{*}(\epsilon)$ be the smallest odd integer such that every series-parallel graph $G$ of odd girth at least $f^{*}(\epsilon)$ has $\chi_{c}(G) \leq 2+\epsilon$.

Theorem 4.4 For any $0<\epsilon<1, f^{*}(\epsilon)=g^{*}(\epsilon)+1$.

Proof. For $k=0,1, \cdots$, let $s_{3 k}=\frac{4 k+3}{2 k+1}, s_{3 k+1}=\frac{8(k+1)}{4(k+1)-1}, s_{3 k+2}=\frac{4(k+1)+1}{2(k+1)}$. Then $s_{0}, s_{1}, s_{2}, \cdots$ is a strictly decreasing sequence. For any $0<\epsilon<1$, let $i$ be the integer such that $s_{i} \leq 2+\epsilon<s_{i-1}$. Assume $i=3 k$ for some $k \geq 0$. By Theorem 4.3 4.2, $f^{*}(\epsilon) \leq 6 k+3$. By Theorem, $g^{*}(\epsilon)>6 k+1$. Hence $g^{*}(\epsilon) \geq 6 k+2$ and $f^{*}(\epsilon) \leq g^{*}(\epsilon)+1$. Therefore $f^{*}(\epsilon)=g^{*}(\epsilon)+1$. The case $i=3 k+1$ or $i+3 k+2$ can be discusses similarly.

\section{References}

[1] J. Chien and X. Zhu, The circular chromatic number of series-parallel graphs of large girth, J. Graph Theory, 33(2000), 185-198.

[2] M. DeVos, Communication at workshop on flows and cycles, Simon Fraser University, 2000.

[3] A. Galluccio, L. Goddyn and P. Hell, High girth graphs avoiding a minor are nearly bipartite, J. Combinatorial Theory (B), to appear.

[4] L. A. Goddyn, M. Tarsi, and C. Q. Zhang, On $(k, d)$-colorings and fractional nowherezero flows, J. Graph Theory, 28(1998), 155-161.

[5] H. Grötzsch, Ein Dreifarbensatz für dreikreisfreie Netze auf der Kugel, Wissenschaftliche Zeitschrift der Martin-Luther-Universität Halle-Wittenberg, Mathematisch-Naturwissenschaftliche Reihe, 8 (1958/1959), 109-120

[6] P. Hell and X. Zhu, The circular chromatic number of series-parallel graphs, J. Graph Theory, 33(2000), 14-24.

[7] F. Jaeger, On circular flows in graphs, Finite and Infinite Sets (Eger, 1981), Colloquia Mathematica Societatis János Bolyai 37, North Holland, (1984) 391-402.

[8] F. Jaeger, Nowhere-zero flow problems, Selected Topics in Graph Theory 3, (L. W. Beineke and R. J. Wilson eds.), Academic Press, London, (1988) 71-95.

[9] W. Klostermeyer and C.Q.Zhang, $(2+\epsilon)$-coloring of planar graphs with large odd girth, J. Graph Theory, 33(2000), 109-119.

[10] D. Moser, The star chromatic number of planar graphs, J. Graph Theory, 24 (1997), $33-43$ 
[11] Z. Pan and X. Zhu, Circular chromatic number of series-parallel graphs of large odd girth, Discrete Mathematics, to appear.

[12] Z. Pan and X. Zhu, Tight relation between the circular chromatic and girth of seriesparallel graphs, manuscript, 2000.

[13] J. Nešetřil and X. Zhu, On Bounded Tree-Width Duality of Graphs, J. Graph Theory, 23 (1996), 151-162

[14] E. Steffen and X. Zhu, On the star chromatic numbers of graphs, Combinatorica, 16(1996), 439-448.

[15] C. Thomassen, Grötzsch's 3-colour theorem and its counterparts for torus and the projective plane, J. Combinatorial Theory (B) 62 (1994), 268-279

[16] W. T. Tutte, A geometrical version of the four color problem, Combinatorial Mathematics and its Applications, (eds. R.C.Bose and T.A.Dowling), University of North Carolina Press, Chapel Hill, (1967).

[17] W. T. Tutte, A contribution on the theory of chromatic polynomial, Canad. J. Math., 6(1954), 80-91.

[18] A. Vince, Star chromatic number, J. Graph Theory 12 (1988), 551-559.

[19] C.Q.Zhang, Circular flows of nearly eulerian graphs and vertex splitting, manuscript, 2000.

[20] C.Q.Zhang, Integer flows and cycle covers of graphs, Marcel Dekker Inc. New York, (1997).

[21] X. Zhu, Planar graphs with circular chromatic numbers between 3 and 4, J. Combinatorial Theory (B), 76(1999), 170-200.

[22] X. Zhu, Circular chromatic number: a survey, Discrete Mathematics, 229 (1-3) (2001), 371-410. 